\title{
A disaster never comes alone: total ostial occlusion of the left main coronary artery with an anomalous origin
}

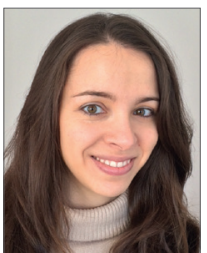

Patrícia Rodrigues*, MD; André Luz, MD; Maria João Sousa, MD; Bruno Brochado, MD; Inês Silveira, MD; João Silveira, MD; Luísa Caiado, MD; Paulo Palma, MD; Henrique Carvalho, MD, PhD; Severo Torres, MD

Cardiology Department, Centro Hospitalar do Porto, Porto, Portugal

This paper also includes supplementary data published online at: http://www.pcronline.com/eurointervention/97th_issue/291

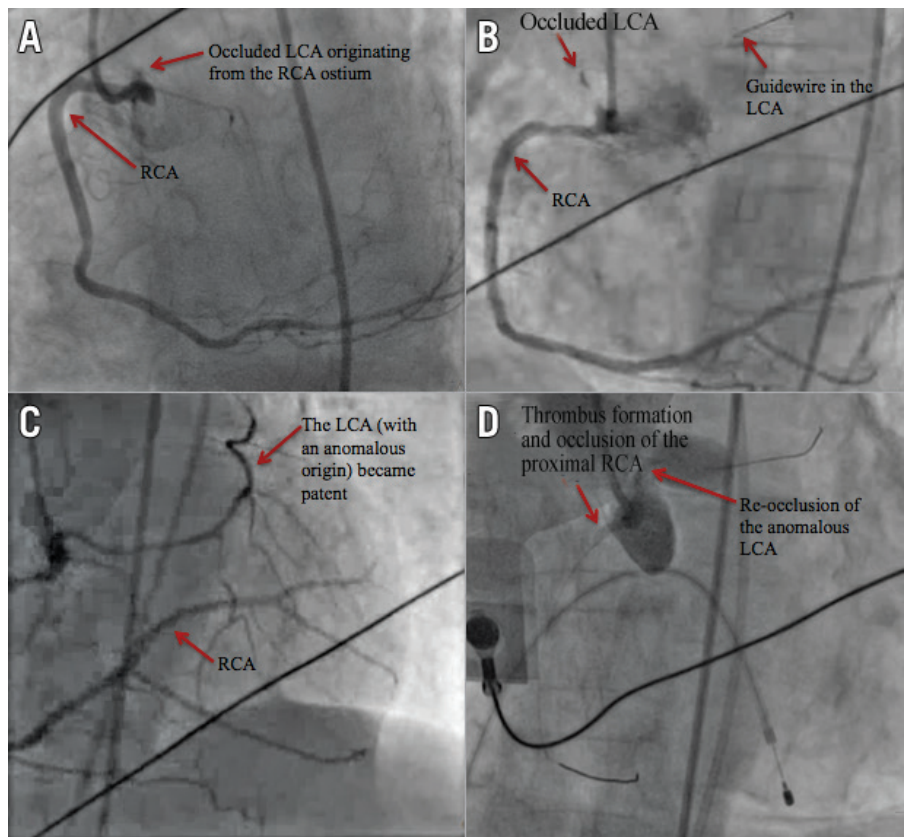

$L C A$ : left coronary artery (left main artery); RCA: right coronary artery

A 68-year-old female presented with chest pain which had started three hours previously, at her father's funeral. The ECG showed ST elevation in the anterior leads and she rapidly evolved into shock. The coronary angiogram showed no lesions in the right coronary artery (RCA) (Moving image 1). However, even after many attempts and angiography of the ascending aorta (Moving image 2), the left coronary artery (LCA) could not be identified. By then, she was in refractory cardiogenic shock and under mechanical ventilation. Finally, the origin of the LCA could be seen rising from the RCA ostium, with a very proximal total occlusion (Panel A, Moving image 3). A guidewire was passed through the lesion (Panel B, Moving image 4), thrombectomy was performed and the LCA became patent (Panel C, Moving image 5). However, massive thrombus and no-reflow then occurred in the RCA (Moving image 6), followed by a total re-occlusion of the LCA (Panel D, Moving image 7). Even after aspiration thrombectomy and glycoprotein IIb/IIIa inhibitor administration, both arteries remained occluded (Moving image 8) and the patient died. Difficulty in angiographic visualisation of the coronary circulation should raise the suspicion of aberrant coronary anatomy. Anomalous origin of the LCA, with a single right coronary ostium, is extremely rare and has been associated with sudden death. Acute total occlusion of an anomalous left coronary, in the context of cardiogenic shock and a thrombogenic environment, turned this case into a nightmare for any interventional cardiologist and a fatal outcome was, unfortunately, not surprising.

\section{Conflict of interest statement}

The authors have no conflicts of interest to declare.

\section{Supplementary data}

The legends of the Moving images can be found in the online version of the paper.

\footnotetext{
* Corresponding author: Avenida da Boavista, 1203-7º D, 4100-130 Porto, Portugal.

E-mail:pfdrodrigues@gmail.com
} 


\section{Supplementary data}

Moving image 1. Right coronary artery (RCA), without any significant stenosis.

Moving image 2. Angiography of the ascending aorta, attempting to visualise the left main artery, which was still not clearly identified.

Moving image 3. Finally, the proximally occluded left coronary artery (LCA) can be seen arising from the RCA ostium.

Moving image 4. A guidewire was inserted into the LCA, crossing the obstruction and finally showing the trajectory of the artery. Moving image 5. After aspiration thrombectomy, the anomalous LCA became patent.
Moving image 6. Thrombus started to form in the proximal RCA. Moving image 7. Even after aspiration thrombectomy and injection of glycoprotein IIb/IIIa inhibitors, there was extensive thrombus and no-reflow in the RCA, followed by a total re-occlusion of the proximal LCA.

Moving image 8. Last recorded moving image, with the patient in cardiac arrest, showing total proximal occlusion of both coronary artery trees. 